\title{
Flight Testing of Novel Compliant Spines for Passive Wing Morphing on Ornithopters
}

\author{
Aimy Wissa ${ }^{1}$ and Nelson Guerreiro ${ }^{1}$ \\ University of Maryland, National Institute of Aerospace, Hampton, VA, 23666 \\ Jared Grauer ${ }^{2}$ \\ Langley Research Center, Hampton, VA 23681 \\ Cornelia Altenbuchner ${ }^{1}$, James E. Hubbard Jr. ${ }^{3}$ \\ University of Maryland, National Institute of Aerospace, Hampton, VA, 23666 \\ Yashwanth Tummala $^{4}$, Mary Frecker ${ }^{5}$ \\ Pennsylvania State University, University Park, PA, 18602 \\ and \\ Richard Roberts ${ }^{6}$, \\ Wright Patterson Air Force Base, Dayton, OH, 45433
}

\begin{abstract}
Unmanned Aerial Vehicles (UAVs) are proliferating in both the civil and military markets. Flapping wing UAVs, or ornithopters, have the potential to combine the agility and maneuverability of rotary wing aircraft with excellent performance in low Reynolds number flight regimes. The purpose of this paper is to present new free flight experimental results for an ornithopter equipped with one degree of freedom (1DOF) compliant spines that were designed and optimized in terms of mass, maximum von-Mises stress, and desired wing bending deflections. The spines were inserted in an experimental ornithopter wing spar in order to achieve a set of desired kinematics during the up and down strokes of a flapping cycle. The ornithopter was flown at Wright Patterson Air Force Base in the Air Force Research Laboratory Small Unmanned Air Systems (SUAS) indoor flight facility. Vicon motion tracking cameras were used to track the motion of the vehicle for five different wing configurations. The effect of the presence of the compliant spine on wing kinematics and leading edge spar deflection during flight is presented. Results show that the ornithopter with the compliant spine inserted in its wing reduced the body acceleration during the upstroke which translates into overall lift gains.
\end{abstract}

\footnotetext{
${ }^{1}$ Graduate Student, Morpheus Laboratory, University of Maryland, National Institute of Aerospace, Hampton, VA 23666, Member AIAA

${ }^{2}$ Research Engineer, Dynamic Systems and Controls Branch, MS 308, National Aeronautics and Space Administration (NASA), Langley Research Center, Hampton, VA 23681 Member AIAA

${ }^{3}$ Langley Distinguished Professor, Morpheus Laboratory, University of Maryland, National Institute of Aerospace, Hampton, VA 23666, Fellow AIAA

4 Graduate Student, Mechanical and Nuclear Engineering Department, The Pennsylvania State University, University Park, PA 16802, Member ASME

${ }^{5}$ Professor of Mechanical Engineering, Mechanical and Nuclear Engineering Department, The Pennsylvania State University, University Park, PA 16802

${ }^{6}$ Research Scientist, Air Force Research Laboratory (AFRL), Wright Patterson Air Force Base, Dayton, OH 45433, Member AIAA
} 


\section{Introduction}

T $\mathrm{N}$ recent years, flapping wing Small Unmanned Aerial Vehicles (SUAVs), or ornithopters, have shown 1 the potential for advancing and revolutionizing SUAV performance in both the civil and military sectors ${ }^{1}$. An ornithopter is unique in that it can combine the agility and maneuverability of rotary wing aircraft with excellent performance in low Reynolds number flight regimes. These traits could yield optimized performance over multiple mission scenarios. Nature achieves such performance in birds using wing gaits that are optimized for a particular flight condition ${ }^{2,3}$.

State of the art designs for wing morphing in flapping wing SUAVs utilize rigid-link mechanisms or involve active morphing techniques, such as rigid four-bar mechanisms ${ }^{4-6}$. In contrast to rigid-link mechanisms and active approaches, the focus of the current research is on the implementation of a novel passive morphing technique using compliant mechanisms. When compared to active morphing, passive morphing mechanisms require no additional energy expenditure, minimal weight addition and complexity. Moreover, there is minimal phase lead/lag between the flapping and the morphing mechanisms, as the morphing is only due to the aerodynamic loads experienced by the ornithopter during flight.

The overall research goal is to passively increase range and endurance of flapping wing SUAVs by bio-inspired structural modifications. The key activities in achieving this goal are as follows:

1) Defining an architecture for passive morphing using a compliant mechanism.

2) Developing a design optimization algorithm for the compliant mechanism.

3) Fabricating and integrating the compliant mechanism into a test ornithopter

4) Comparing the performance of the ornithopter with and without the compliant mechanism to investigate its effect on the steady level flight performance.

In this paper, recently collected free flight data comparing performance metrics for test ornithopters with and without the compliant mechanism inserted in their wings' leading edge spars are presented. The data collected were used to assess the effect of the compliant mechanism on wing kinematics. Wing kinematics data were previously collected on a bench top where the ornithopter fuselage was clamped to a six degree of freedom (DOF) load cell ${ }^{7,8}$. The purpose of the flight test described in this paper is to understand the effect of the compliant mechanisms during free flight and to determine whether the previous bench test performance relates directly to free flight performance, as well as to increase the understanding of the flight physics and dynamics of flapping wing unmanned vehicles. This paper is organized as follows: the research approach and a summary of the compliant spine design optimization is presented in Section II. Section III then discusses the experimental procedures and ornithopter set-up. Lastly Sections IV shows the free flight tests wing kinematics and leading edge deflection results.

\section{A. Bio-inspired Approach}

\section{Methodology}

The benefits and efficacy of passive wing morphing attained by introducing an asymmetry into the leading edge wing spar during the up and down strokes have been investigated ${ }^{9,10}$. Billingsely et al. installed passive torsional springs in the leading edge spar at the wing half span to exploit the advantages of wing surface area reduction during the upstroke $\mathrm{e}^{10}$. These springs were designed to deflect on the upstroke only and lock during the downstroke. Wing bending during the upstroke reduces the wing 
relative area (i.e., the wing area perpendicular to the flapping motion), which in turn mitigates the drag penalties and negative lift experienced by the test ornithopter during this portion of its wing beat cycle.

While the results of Billingsely's experiment showed an increase in net lift, there were also significant thrust penalties. It was concluded that more sophisticated wing kinematics are required in order to maintain the lift gains while mitigating thrust penalties thus improving the overall aerodynamic performance of the ornithopter. The desired kinematics can be found in natural avian flyers. A bioinspired gait known as the Continuous Vortex Gait $(\mathrm{CVG})$ is shown in Figure $1^{11}$. A detailed discussion of the kinematics of CVG can be found in References 2 and 3. The advantage of using the CVG is that it is an avian gait that can be implemented passively because it requires motion in only one major joint, namely the wrist.

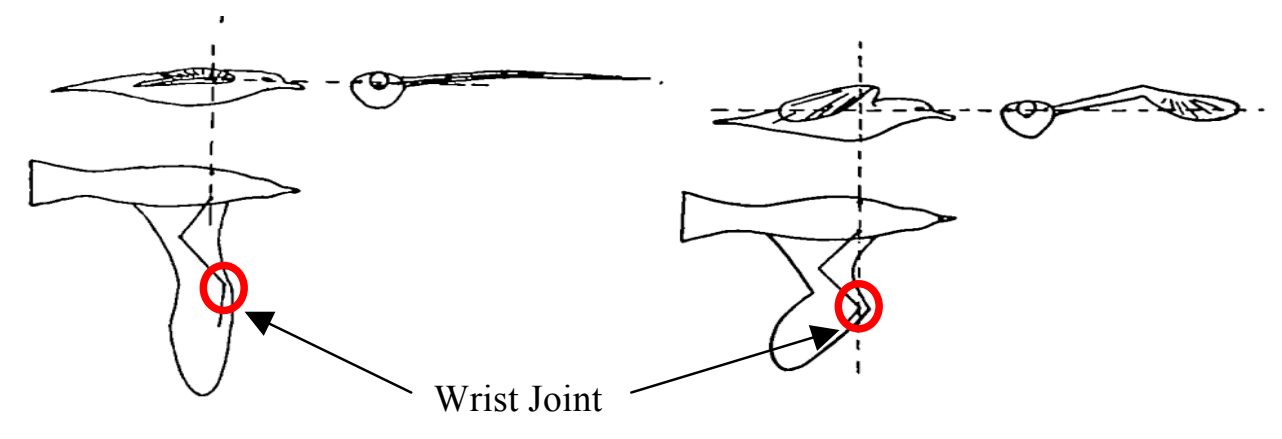

Figure 1. During the continuous vortex gait the wings are fully extended at mid downstroke (left) and bent, twisted and swept at mid upstroke (right). ${ }^{11}$

In order to implement the CVG on a test ornithopter and to achieve improved performance, specific wing kinematics are required. The outer section of the wing has to bend, sweep and twist simultaneously during the upstroke, while remaining fully extended during the downstroke. The wrist, as shown in Figure 1 , is the primary joint responsible for the radical shape changes in the CVG gait. In order to implement the CVG kinematics, a compliant mechanism called a compliant spine was placed in the location where an avian wrist would exist, namely at $37 \%$ of the wing half $\operatorname{span}^{2}$. Figure 2 shows a schematic of the compliant spine and its location along the leading edge spar of an ornithopter.

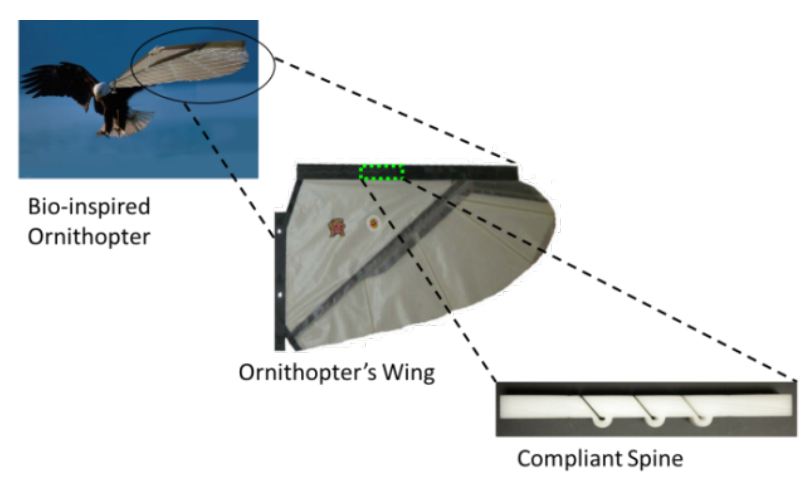

Figure 2. The compliant spine is inserted into the leading edge spar to mimic the function of an avian wrist. 


\section{B. Compliant Spine Design Optimization}

The compliant spine (CS) is a novel monolithic, nonlinear compliant mechanism. Compliant mechanisms have numerous advantages over rigid-link mechanisms. They are easy to manufacture and lower cost than their rigid link counterparts because they are usually monolithic in nature. During the upstroke, the compliant spine must allow the wing to morph in the bending direction; while during the downstroke; it must become stiff, mimicking a rigid spar. A schematic illustrating the desired stiffness of a compliant spine compared to the rigid spar and to a torsional spring is shown in Figure $3(\mathrm{a})^{12}$. The desired stiffness of the compliant spine is nonlinear. It is stiff in the downstroke, similar to a rigid spar, and flexible in the upstroke, similar to a torsional spring. Figure 3(b) shows a CS design with three compliant joints (CJs). Note that this design is flexible in bending during the upstroke because of the compliance of the semi-circular compliant hinges $(\mathrm{CHs})$, and it is stiff in bending during the downstroke because the slanted faces come into contact with one another.

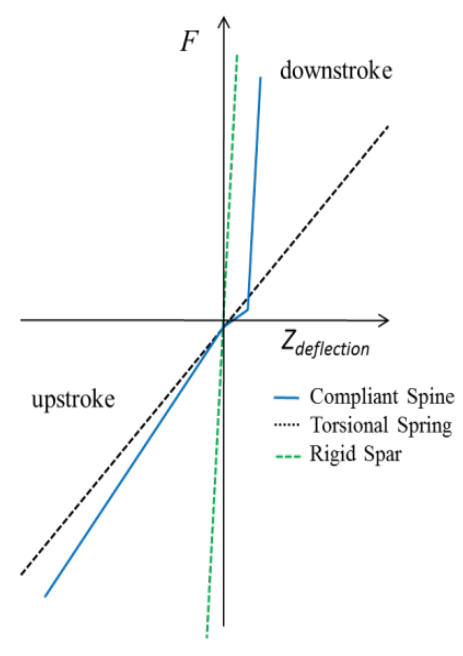

(a)

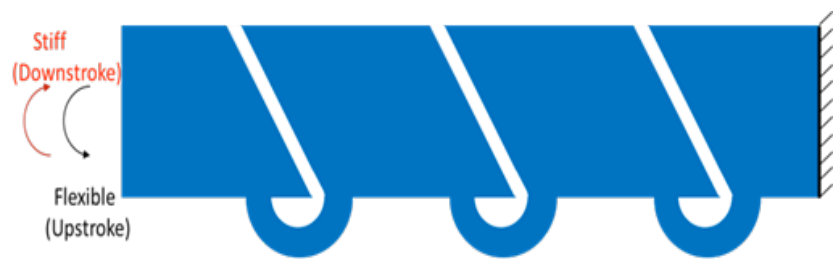

(b)

Figure 3(a) The desired stiffness of the compliant spine is nonlinear. The $Y$-axis represents the forces (F) during a flapping cycle and the $\mathrm{X}$-axis ( $\mathrm{Z}$ deflection) represents the compliant spine tip bending deflection (b) A compliant spine with three compliant joints

An optimization procedure, where the spine was subject to dynamic load analysis, was implemented in order to design a single compliant hinge with a single compliant joint ${ }^{12,13}$. The parameters that affect the performance of a compliant spine are the number of compliant joints and the shape of each compliant joint. The design parameters that affect the joint's stiffness during the upstroke are related to the shape of the compliant hinge, while the design parameters that affect the downstroke stiffness are related to the geometry of the contact surfaces. The design optimization procedure yields a family of designs that meet the requirements for maximum von-Mises stress, and desired wing bending deflections, thus several CS were tested during the flight test.

Different loading conditions were used during the optimization to simulate lift forces acting on the compliant spines. Lift force distribution was approximated as either a tip load or a bending moment applied at the tip of the CS. The magnitude of the pure moment applied on the CSs is equal to the maximum moment that the CSs saw when a tip load, representing the integrated lift load ${ }^{12,13}$, was applied 
on them. In reality, the CSs experience a lift load distribution that is a combination of both moment and bending loads. The design optimization procedure is described in detail in References 12 and 13.

\section{Experimental Procedures}

Several flights tests were performed to assess the performance of the various CS designs. The flight tests were conducted at Wright Patterson Air Force Base (WPAFB) in the Air Force Research Lab's (AFRL) Indoor Small Unmanned Aerial Systems (SUAS) laboratory. This is the largest Vicon motion capture system lab in the United States. This section explains the equipment used, ornithopter preparation, test configurations flown and the experimental set-up. Figure 4 shows a picture of the test platform in the flight lab.

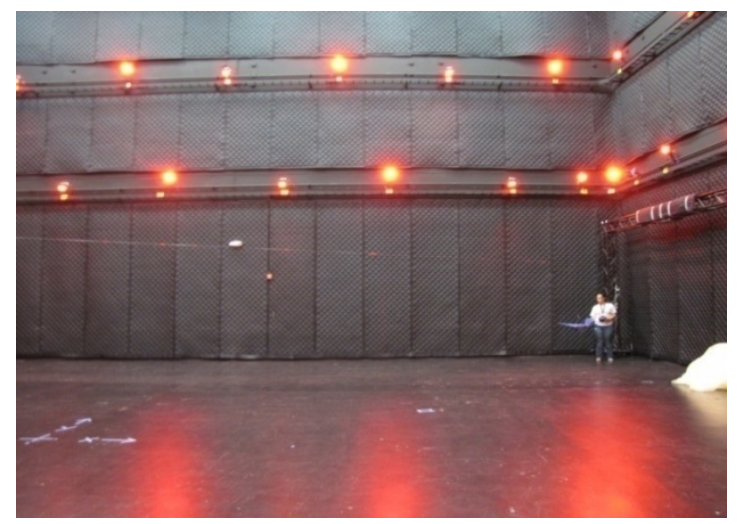

Figure 4. Test platform in the AFRL SUAS flight lab. The facility includes 60 motion capture cameras and is considered the largest in the U.S.

\section{A. Equipment and Facilities}

The AFRL SUAS indoor flight test laboratory is composed of an enclosed flight test chamber and a control room. The test chamber is a large, instrumented room where vehicles can be flown. It is roughly

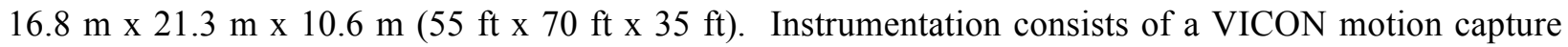
system with 60 motion capture cameras. By adding small retro-reflective markers to a vehicle, the VICON system can track position and orientation of the vehicle with an accuracy of about $1.0 \mathrm{~mm}$. The control room is used to simultaneously command test vehicles as well as to process and record test data such as vehicle position/orientation, velocity, acceleration, commands, sensor telemetry, and video-stream and audio data.

\section{B. Ornithopter Setup}

Fifty-three reflective ball markers were attached on the test vehicle in locations that were necessary in order to obtain enough data to fully understand the wing kinematics and body dynamics. Forty-four 6.35 $\mathrm{mm}(0.25 ")$ diameter markers were placed in an asymmetrical pattern in order to aid in tracking. The other 9 markers were distributed as follows: 5 were placed on the fuselage to determine the ornithopter's body dynamics, 3 were placed on the tail to record user control inputs and 1 was placed at the wing root to measure the wing angle during a given flapping cycle. The Vicon ${ }^{\circledR}$ motion capture system was used to capture and contrast the wing 3D kinematics of the ornithopter with and without the compliant spine inserted in the leading edge spar. Figure 5 shows the placement of the wing reflective markers. Data was collected at $200 \mathrm{~Hz}$ during these tests and was converted into Comma Separated Values (CSV) format for analysis in MatLab ${ }^{\mathrm{TM}}$. 


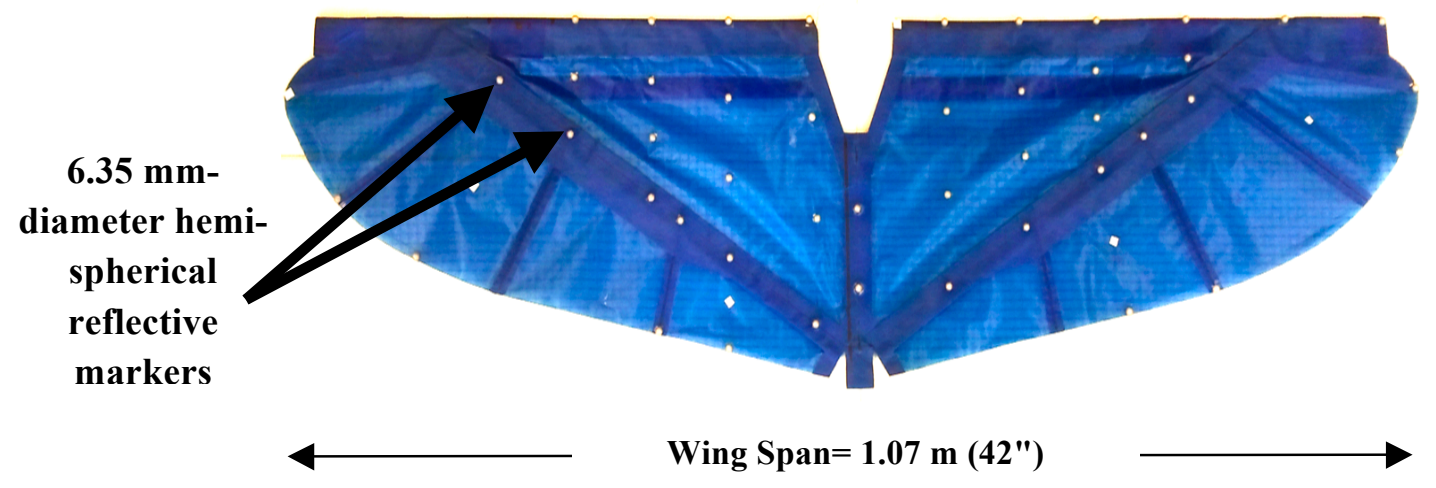

Figure 5. Wing Reflective Markers Placement. Markers were distributed over both wings to balance the weight and they were placed asymmetrically to aid with tracking and post processing.

\section{Test Setup}

To aid in repeatability and prevent impacts, a low-friction tether was utilized to guide the vehicle in the test chamber. The vehicle was suspended from a lead wire off this tether in order to constrain its flight path. The lead line was able to slide along the tether by using a barrel swivel. The tether was strung horizontally between two trusses at opposite corners of the flight lab at $2.1 \mathrm{~m}(7 \mathrm{ft})$ height in order to maximize flight distance and keep it at a height where camera coverage is ideal. The tether also prevented the vehicle from leaving the region where high speed cameras were recording the flight. A wire crimp and a braking tether were used at the end of the path in order to stop the vehicle. Figure 6 shows a schematic of the test setup.

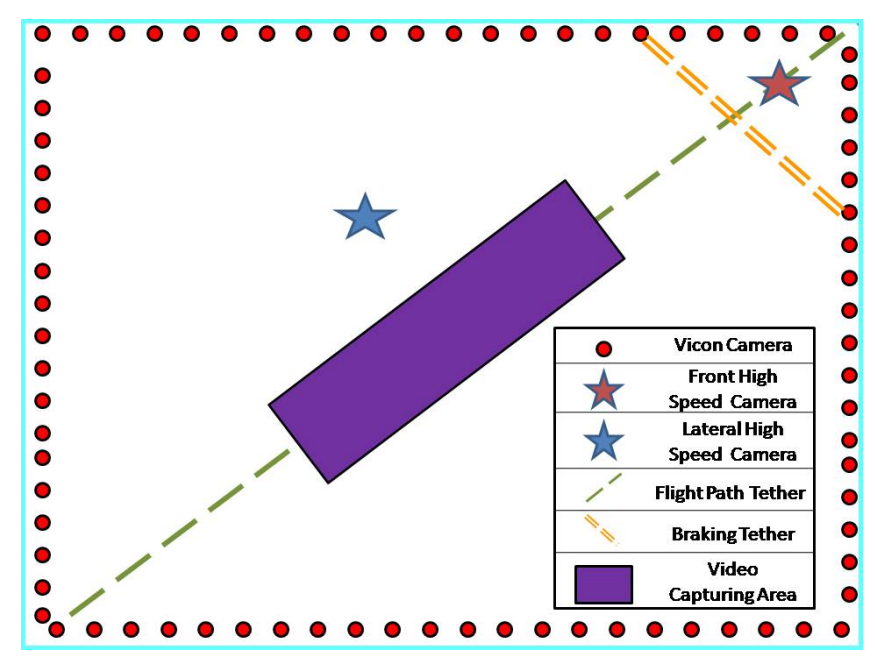

Figure 6. Test Setup Schematic showing the Vicon cameras (representative), high speed cameras, flight path, braking tether, and video capturing area

\section{Ornithopter Configurations}

Five ornithopter wing configurations were tested. The first configuration was the Ornithopter with a uniform, solid, carbon fiber wing spar. The remaining configurations consisted of compliant wing spars, inserted in the leading edge spar at $37 \%$ of the wing half-span to mimic the function of the avian wrist. Four compliant spine designs were tested, thus there was a total of five configurations ( 1 solid and 4 compliant). Table 1 includes common test platform specifications ${ }^{14}$. 
Table 1. Ornithopter specification that are common across all test configurations.

\begin{tabular}{|c|c|c|c|c|}
\hline Span & Max. Chord & Flapping Rate & Speed & Range \\
\hline $1.07 \mathrm{~m}(42 ")$ & $0.28 \mathrm{~m}(11 ")$ & $4-6 \mathrm{~Hz}$ & $2.5-8.5 \mathrm{~m} / \mathrm{s}(8.2-27.9 \mathrm{ft} / \mathrm{sec})$ & $0.8 \mathrm{~km}$ \\
\hline
\end{tabular}

\section{1) Solid Configuration}

Figure 7 shows an image of a Morpheus Lab custom-built test ornithopter (ML 101) with a solid leading edge spar. This model served as the test baseline. The mass of the solid configuration ornithopter including batteries, motor, current sensor, markers, and data loggers was measured to be $528 \mathrm{~g}$.

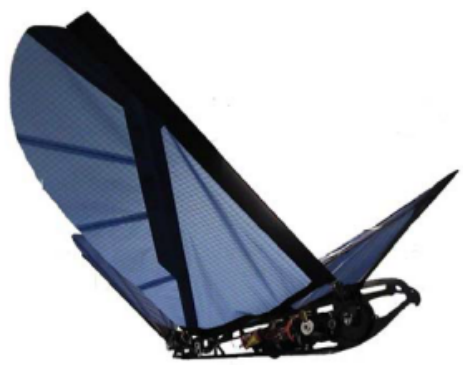

Figure 7. Test ornithopter with a rigid carbon fiber leading edge spar

\section{2) Compliant Configurations}

The compliant ornithopter has the same components as the solid ornithopter with the only difference being that a compliant spine was inserted in the leading edge spar of both its wings. Four compliant spines were tested during this flight test. All the compliant spines were $63.5 \mathrm{~mm} \mathrm{(2.5")} \mathrm{long} \mathrm{with} 25.4$ $\mathrm{mm}(1 ")$ tab on both sides to allow for attachment to the leading edge spar. The compliant spine was attached to the carbon fiber spar using six 5-40 bolts and a Delrin collar, as shown in Figure 8. The major differences between the various compliant spine designs are the number of compliant hinges and the compliant joint geometry, which resulted from the various loading conditions applied during their design optimization process.

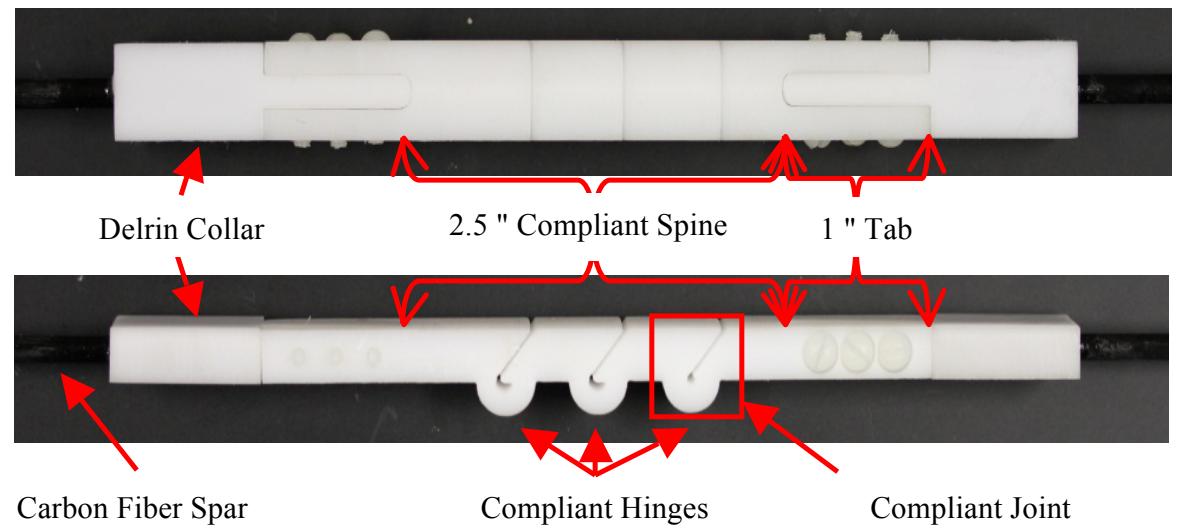

Figure 8. Compliant spine assembly components

Each compliant spine design was given a name consisting of a number and two letters (e.g. 4TL). The number in the name of the compliant spine refers to the design number assigned by the optimization algorithm. The two letters in the name refer to the loading condition under which this compliant spine was 
designed. TL stands for tip load and PM stands for pure moment. Figures 9a and b show the tip loading and pure moment loading condition, respectively, as well as the boundary conditions.

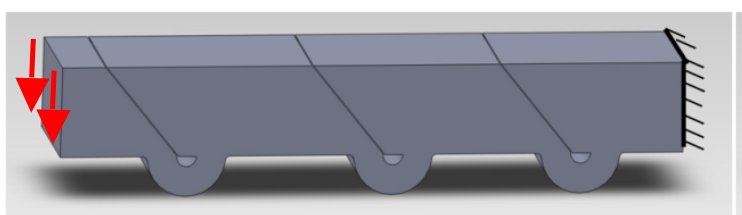

(a)

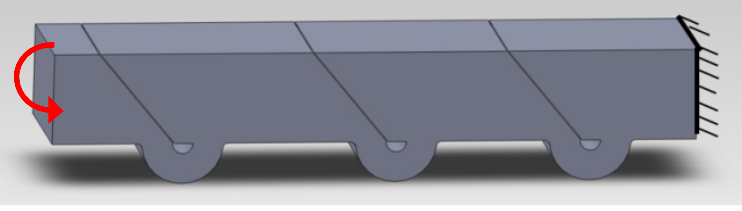

(b)

Figure 9. Compliant spines were assumed to have clamped-free boundary condition and were designed using either (a) tip load (TL) loading condition or (b) pure moment (PM) loading condition.

Table 2 lists the compliant spine designs, their loading condition, the number of compliant hinges, and the total mass of the ornithopter with these spines inserted in its leading edge and including all the components that was previously present in the solid configuration. Design 4TL was previously tested during the bench test ${ }^{7}$ and therefore was included in the free flight test to aid in the comparison between the bench and free flight testing results. Table 3 shows all four compliant spine designs and their predicted stress distribution at mid upstroke.

Table 2. Specifications of compliant spine designs

\begin{tabular}{|c|c|c|c|}
\hline Design Name & $\begin{array}{c}\text { Loading } \\
\text { Condition }\end{array}$ & $\begin{array}{c}\text { Number of } \\
\text { compliant joints }\end{array}$ & Ornithopter Mass \\
\hline Comp 4TL & Tip load & 3 & $614 \mathrm{~g}$ \\
\hline Comp 4PM & Pure moment & 2 & $611 \mathrm{~g}$ \\
\hline Comp 14PM & Pure moment & 3 & $614 \mathrm{~g}$ \\
\hline Comp 24PM & Pure moment & 4 & $612 \mathrm{~g}$ \\
\hline
\end{tabular}


Table 3. The geometry (2nd column) and von-Mises stress distribution at mid upstroke (3rd column) of the four compliant spine configurations

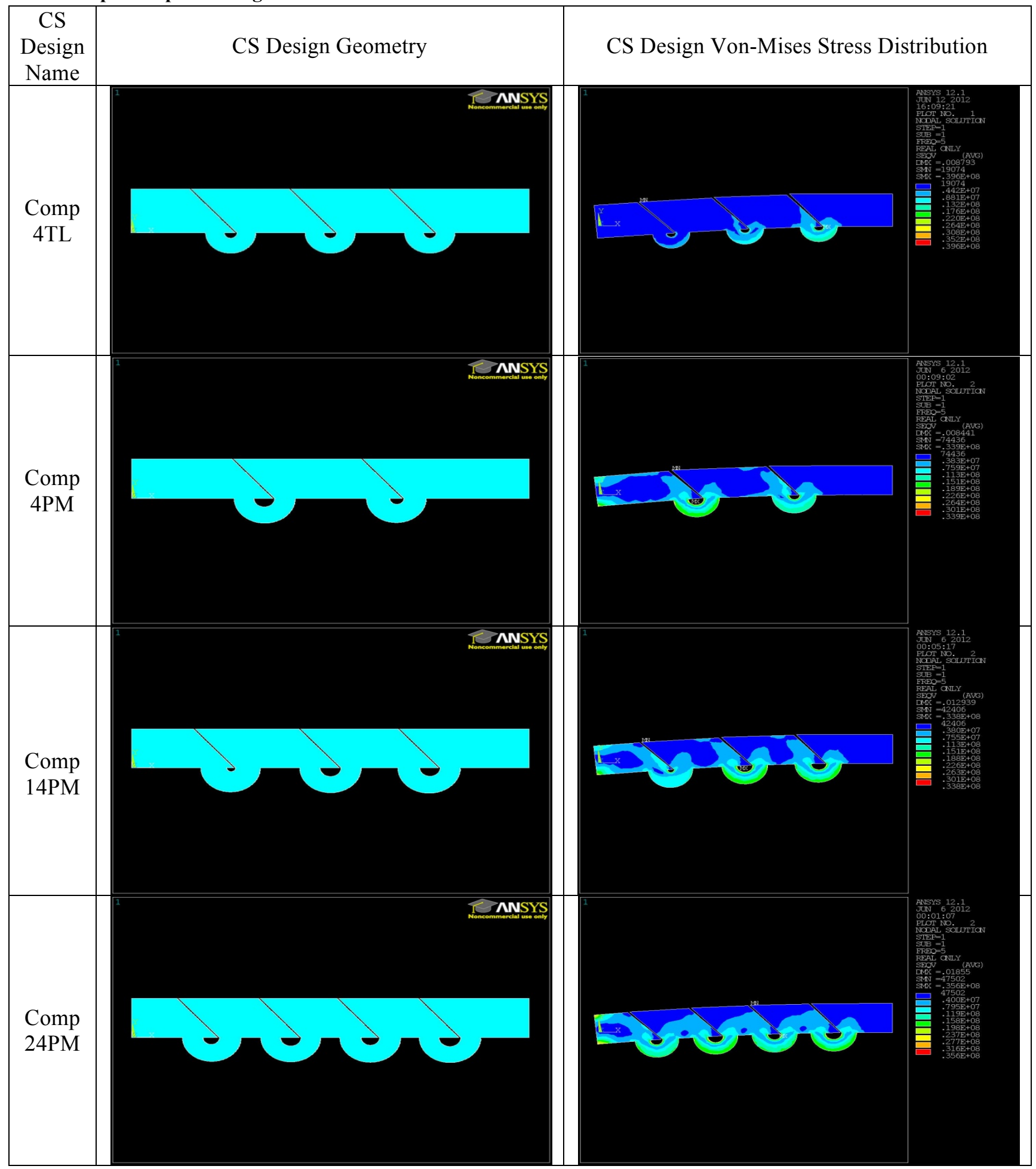




\section{Results}

This section discusses the ornithopter's overall free flight dynamics as well as the effect of the presence of the compliant spine on: a) the acceleration of the center of mass, and b) the leading edge spar deflections of the test ornithopter during one wing cycle.

\section{A. Ornithopter's Free Flight Dynamics}

Before the completion of the aforementioned flight test, literature describing measurement of detailed free flight kinematics of avian scale ornithopters was very limited. One of the goals of this flight test was to extend the current database of steady level, free flight data. In order to attain this goal, consistent and repeatable kinematics over several flapping cycles are required. Figure 10 shows the measured X, Y and $\mathrm{Z}$ position of the 53 markers that were mounted on the ornithopter. The figure also illustrates that good tracking was achieved. The plot shows over eight consistent flapping cycles and $1.5 \mathrm{sec}$ of flight data. Not only was it required that consistent and repeatable kinematics were achieved, but also this data needed to be recorded during steady level free flight. Figure 11 shows the altitude above ground level (AGL) of the center mass of the ornithopter's fuselage. The location and orientation of the fuselage center of mass was calculated by fitting a rigid body to the fuselage using the five markers that were mounted on it. More details about this technique can be found in reference 15. The black dotted line in the figure represents a threshold altitude above which the vehicle is no longer hanging on the tether and therefore in free flight. Figure 11 shows that the ornithopter was flying well above this threshold altitude and therefore this flight test was successful in measuring and recording the ornithopter's kinematics over several flapping cycles and during steady level flight.

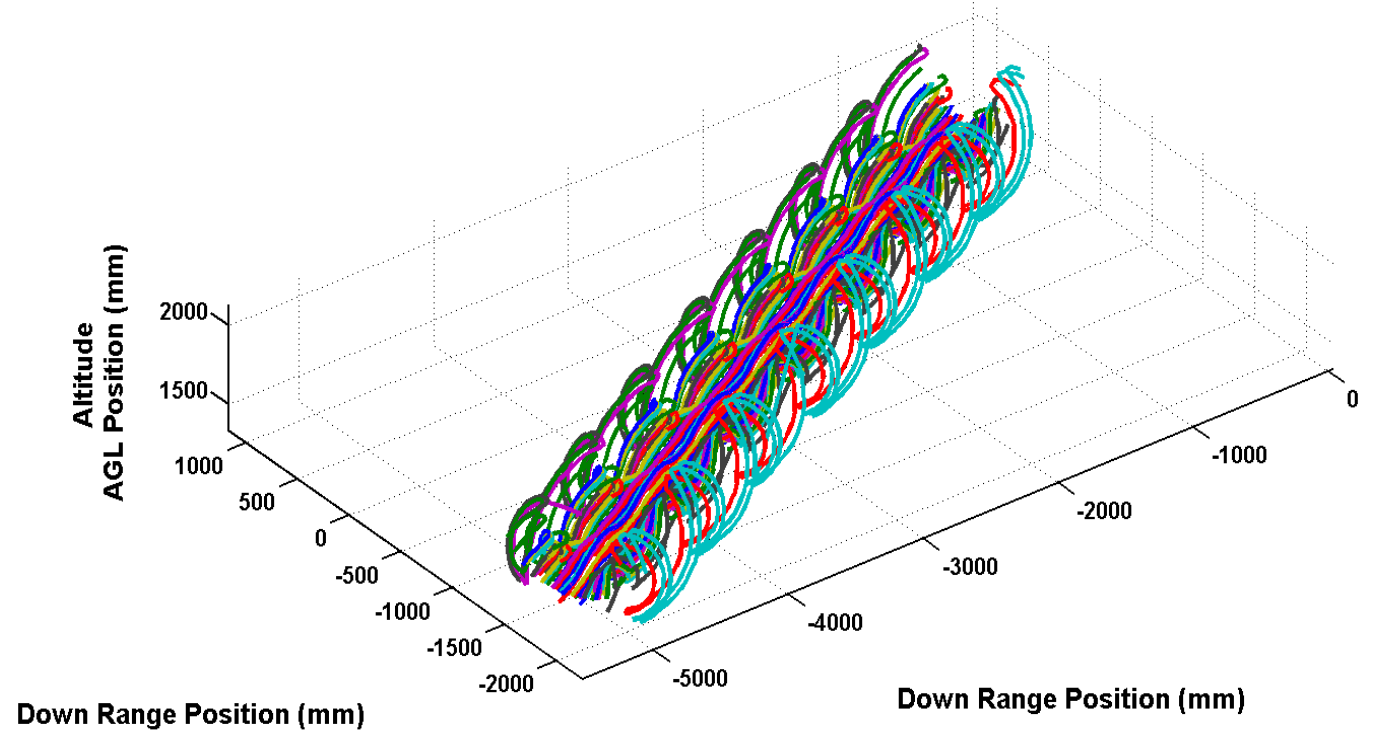

Figure 10. $\mathrm{X}, \mathrm{Y}$, and $\mathrm{Z}$ position of the 53 markers mounted on the ornithopter showing over eight flapping cycles of consistent and repeatable kinematics. The $X$ and $Y$ position represents the down range location of the marker and the $Z$ position represent the marker's altitude above ground. 


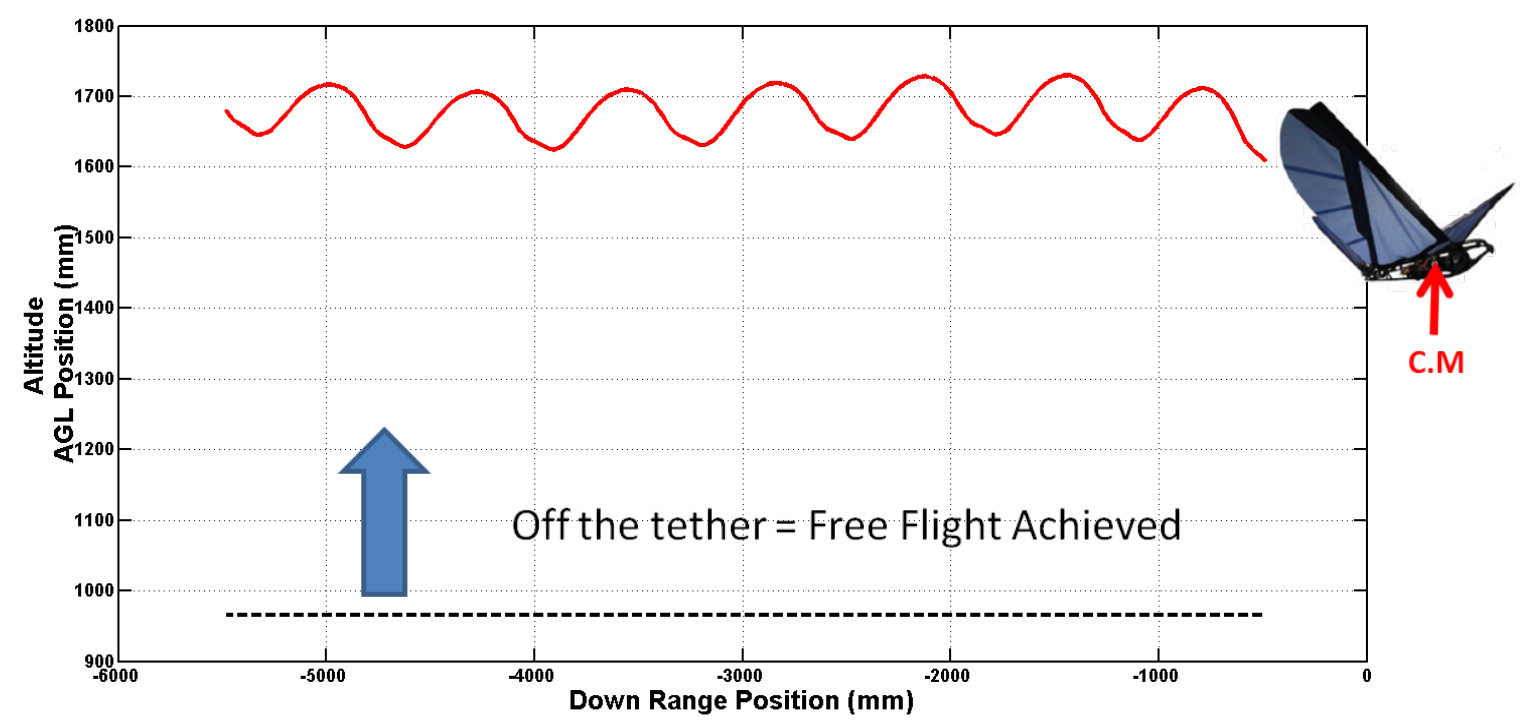

Figure 11. Ornithopter fuselage center of mass altitude (solid red line) showing that the test ornithopter is flying above the altitude threshold (dotted black line) for free flight .

After establishing free flight, the ornithopter dynamics was examined. Figure 12 compares the altitude above ground level of the right wing tip marker and the fuselage's center of mass for 3 flapping cycles. The figure shows that the ornithopter's body position is out of phase with the wing tip position; in other words as the wing flaps downwards, the fuselage is moving upwards.

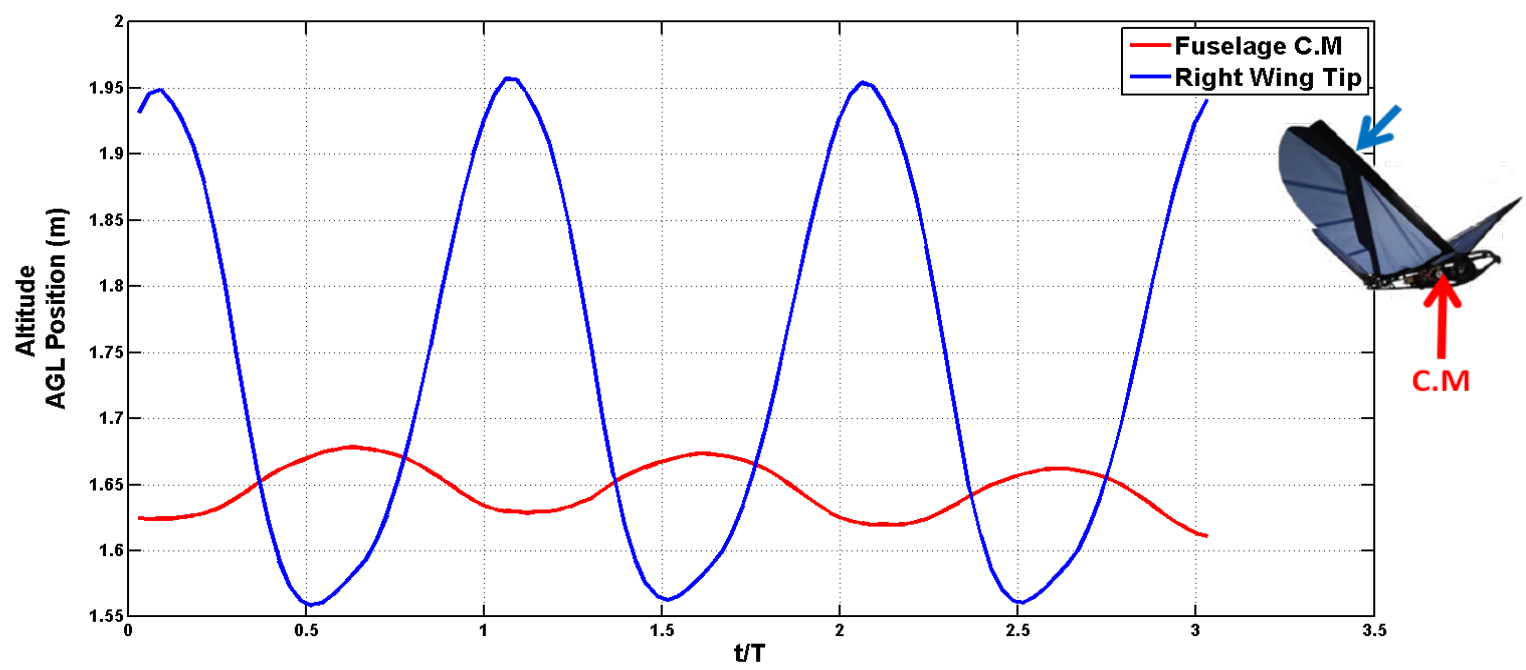

Figure 12. The altitude above ground level of the right wing tip marker (blue) and the fuselage's center of mass (red) versus time normalized by the period of one flapping cycle

Figure 12 also shows the ornithopter's body dynamics; an aspect of the vehicle's flight physics that is only possible to monitor through free flight testing. In order to further understand the body dynamics, the position of the fuselage center of mass was differentiated twice to obtain the acceleration. Figure 13 shows the wing tip $\mathrm{Z}$ position with respect to the center of mass, and the center of mass acceleration. Note for Figures 13 through 17, negative is upwards and positive in downwards. 


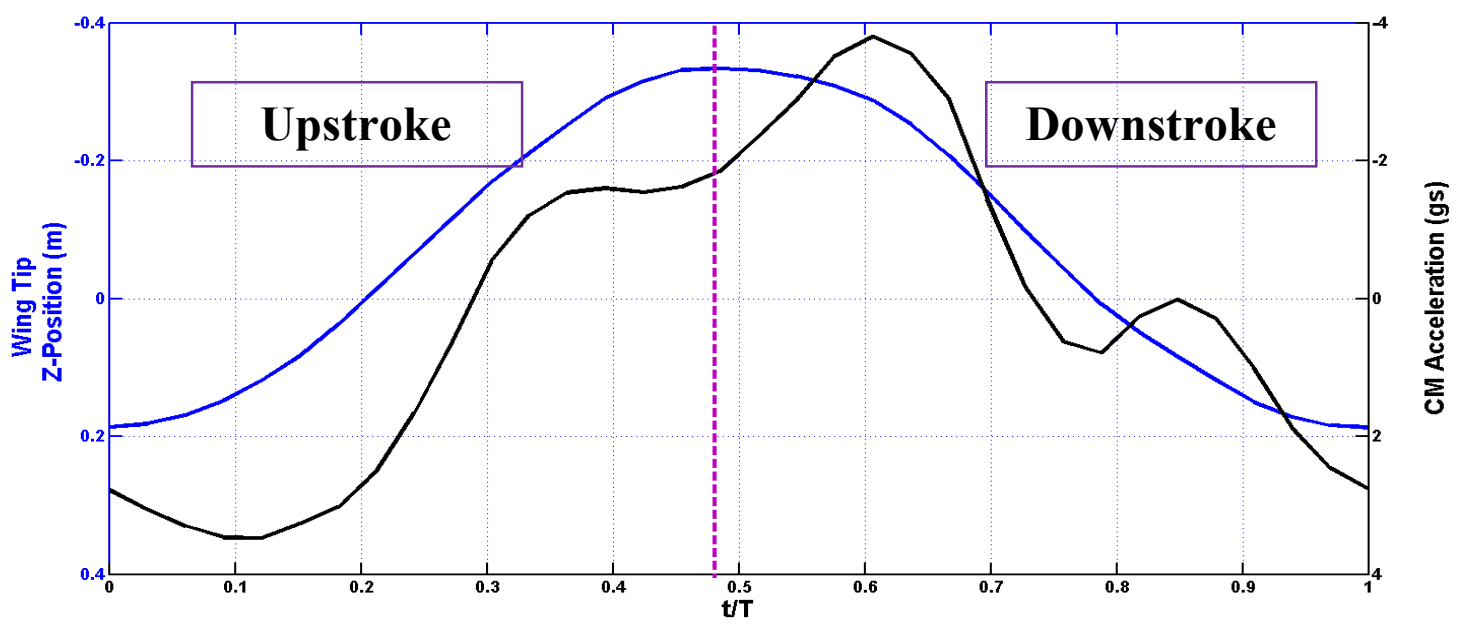

Figure 13. Wing tip marker Z-position with respect to the center of mass (blue) and the center of mass acceleration (black) versus time normalized by the period of one flapping cycle

The significance of the body dynamics is apparent in Figure 13. The ornithopter body has an acceleration of \pm 4 gs. During prior $^{15}$ and the current flight tests, it was noticed that the vertical acceleration or load curves had a peak after the upstroke-downstroke transition and downstroke-upstroke transition points, as shown in Figure 14. Prior to these flight tests the reason for this peak remained unexplained. Figure 14 shows that the peak that occurs after the transition points is due to the thrust flap portion of the wing changing directions. The thrust flap portion of the wing is shown in Figure 15 and it is the part of the wing primarily responsible for the thrust production. The thrust flap lags the main wing leading edge spar in changing directions at the stroke transition points. The thrust flap can be thought of as a hinged flat plate, as it changes direction, it causes an increase in the body's acceleration.

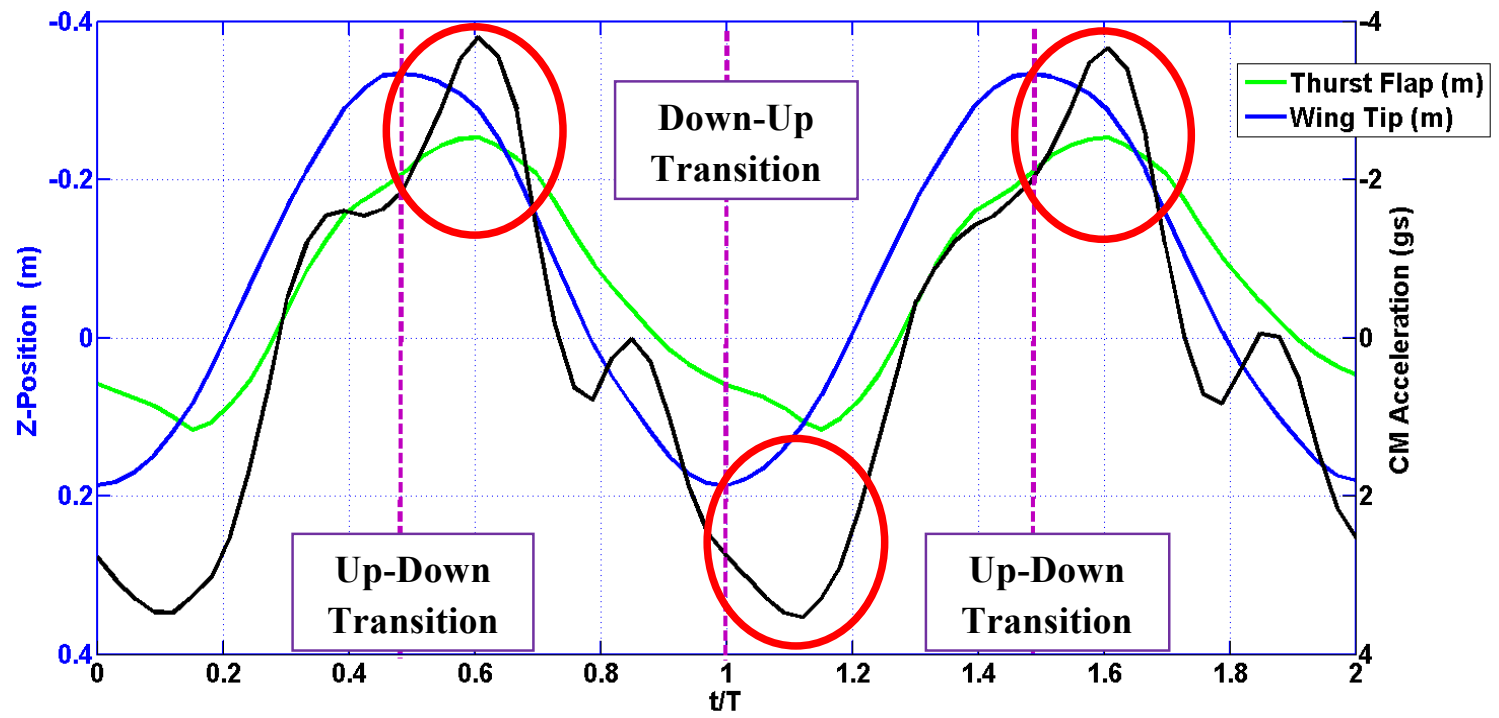

Figure 14. Wing tip marker Z-position with respect to the center of mass (blue), thrust flap marker Zposition with respect to the center of mass (green), and the center of mass acceleration (black) versus time normalized by the period of one flapping cycle 


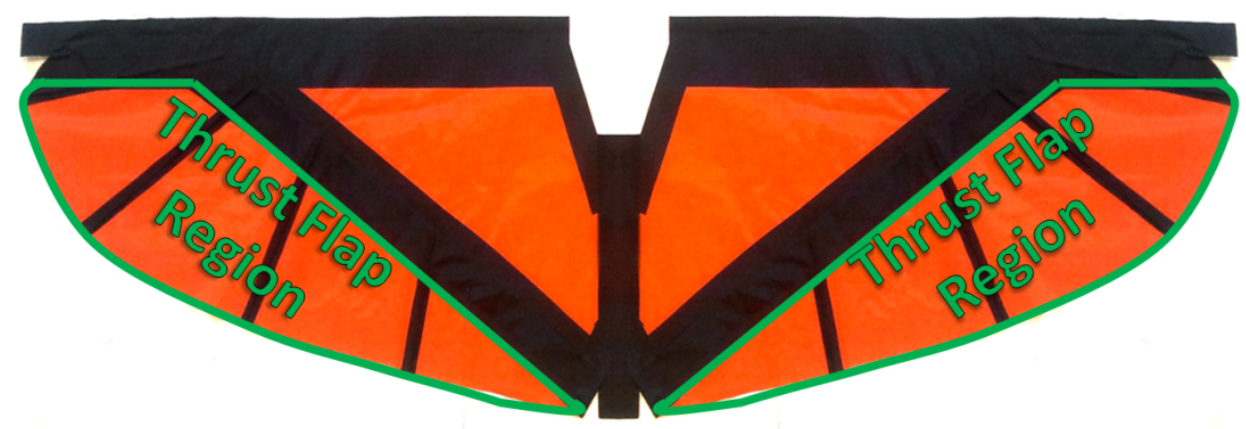

Figure 15. Wing planform showing the thrust flap region. When the thrust flap changes direction a peak occurs in the body vertical acceleration curve.

\section{B. Effect of Compliant Spine Presence}

During the flight test, the four compliant spine designs described in section III.D were inserted into the wing leading edge spar. This section demonstrates the effect of the presence of the compliant spine on the body's vertical acceleration and leading edge spar bending deflection. The compliant spine design optimization results predicted that a compliant spine with more compliant hinges tended to have a greater maximum bending deflection ${ }^{13}$. For this paper, results from designs Comp 24PM and Comp 4PM are shown as these designs have the most and least number of compliant hinges, respectively.

\section{3) Effect on Vertical Body Acceleration}

Figure 16 shows vertical acceleration of the fuselage center of mass for the solid, Comp 24PM, and Comp 4PM configurations versus time normalized by the period of one flapping cycle.

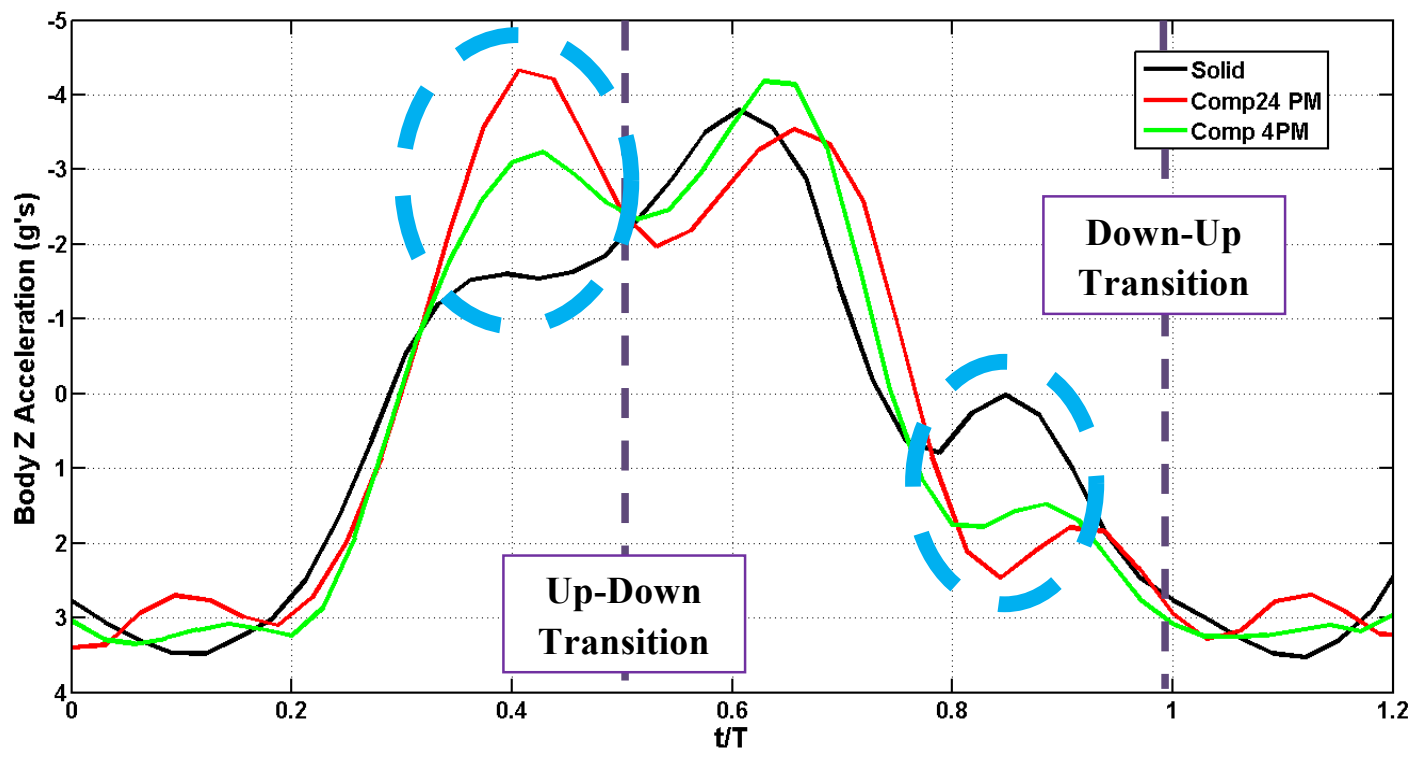

Figure 16. Vertical acceleration of the fuselage center of mass for the solid (black), Comp 24PM (red), and Comp 4PM (green) configurations versus time normalized by the period of one flapping cycle

The effect of the presence of a compliant spine in the leading edge spar is evident at two locations in the flapping cycle, as shown in Figure 16. The first location is at the end of the upstroke right before the upstroke to downstroke transition. At this point, the compliant spine is changing from the bending 
configuration to the locked configuration, creating an effect similar to a whip lash and therefore increasing the body acceleration. Negative acceleration corresponds to upwards acceleration, a higher negative acceleration indicates an increase in the body's upwards acceleration and wings' downward acceleration, or an increase in the amount of positive lift produced by the wings. Design Comp24PM is the most flexible design, resulting in the larger difference from the solid design when compared to design Comp 4PM. During the downstroke portion of the flapping cycle, the behavior of the ornithopter with and without the compliant spine was predicted to be similar because the contact surfaces in each compliant hinge come together. However due to the presence of the contact gaps and the difference in modulus between the material of the solid spar (uni-directional carbon fiber composite) and the material of the compliant spine (Delrin) some undesirable bending occurs during the downstroke. The second location where the effect of the compliant spine is apparent is during the second half of the downstroke. At this point the compliant spine has locked completely and the undesirable upward bending has started to occur. Thus, during the second half of the downstroke, an increase in the positive acceleration is observed due the presence of the compliant spine. Positive acceleration corresponds to downward acceleration, so a higher positive acceleration indicates an increase in the body's downward acceleration and wings' upward acceleration and therefore an increase in the amount of negative lift produced by the wings. Further data analysis shows that when the mean acceleration over one flapping cycle is computed, the ornithopter with Comp 24PM and Comp 4PM inserted in its wing reduced the body's center of mass positive acceleration by $69 \%$ and $5 \%$, respectively. The positive acceleration reduction translates into overall lift gains, which would confirm previous bench test results ${ }^{7}$.

\section{4) Effect on Leading Edge Spar Deflections}

During the upstroke the compliant spine is allowed to bend because of the presence of the compliant joints, while during the down stroke, the contact surfaces come together, locking the compliant spine so that it acts like the uniform carbon fiber spar. Figures $17 \mathrm{a}$ and $\mathrm{b}$ show the bending deflection of the markers placed on the right wing leading edge spar at mid upstroke and mid downstroke. 


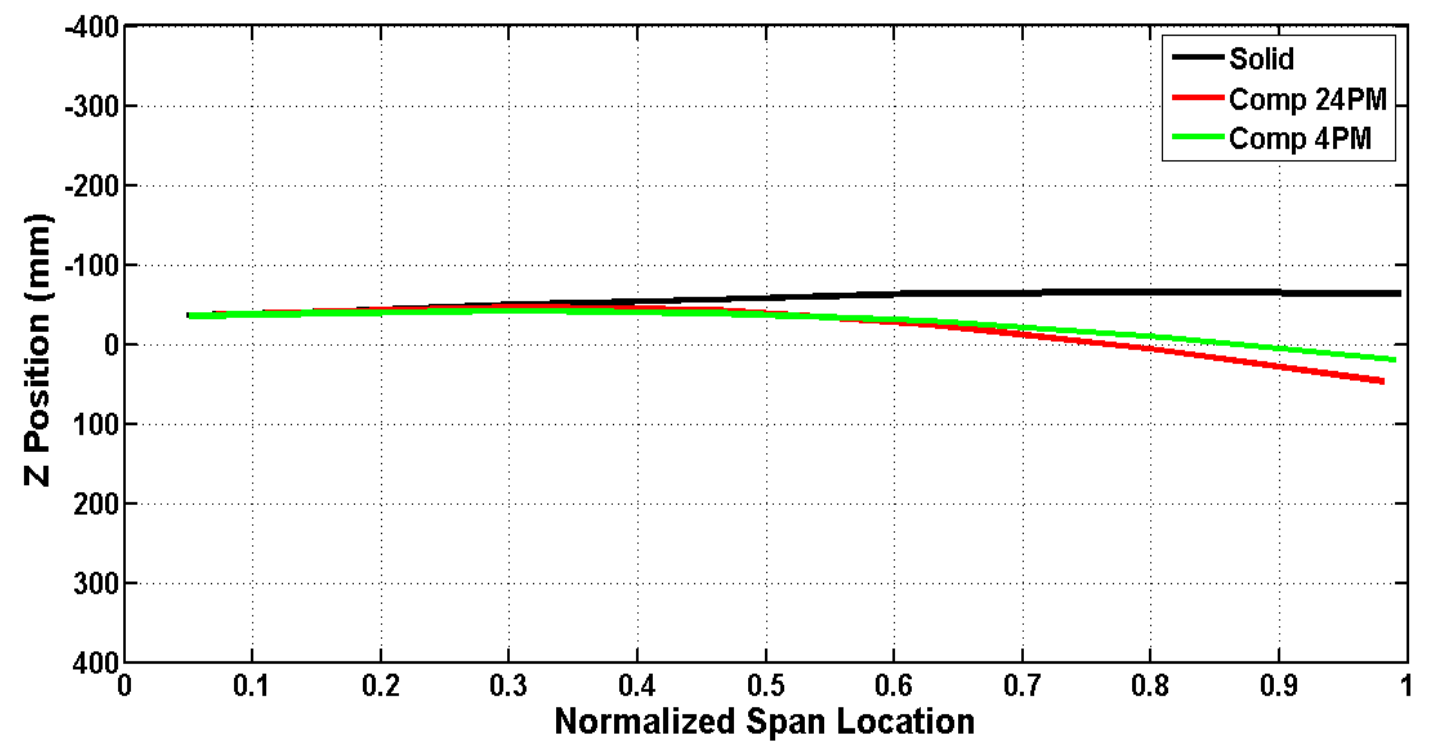

(a) mid upstroke

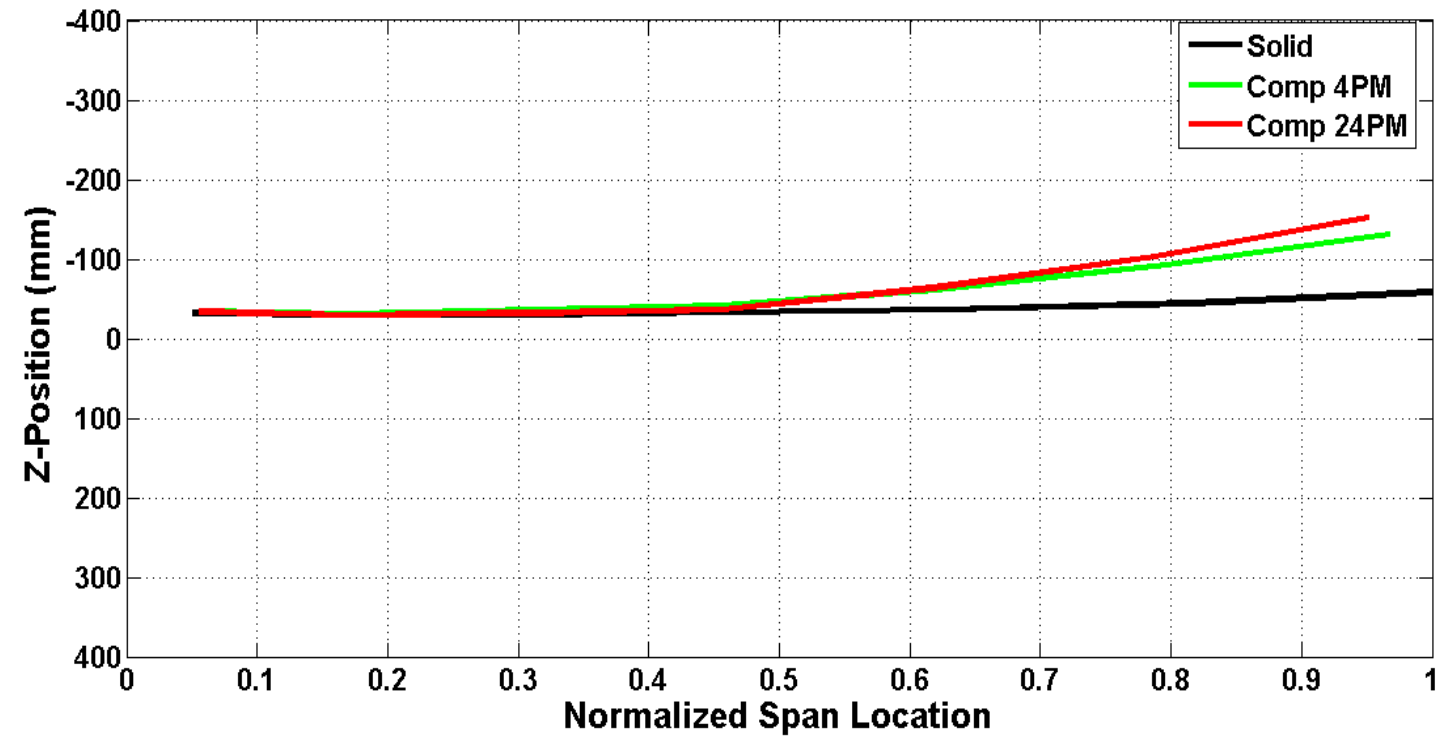

(b) mid downstroke

Figure 17: The $Z$ position of the reflective markers mounted at the right wing leading edge spar versus the normalized span location at (a) mid upstroke and (b) mid downstroke

Figure 17 confirms the design optimization result stating that Comp 24PM is more flexible than design 4PM. Figure 17a shows that the configuration with the compliant spar inserted in its wings is allowed to bend during the upstroke. The relative bending deflection between the compliant spar tip marker and the solid spar tip marker is $110.7 \mathrm{~mm}$ and $83.24 \mathrm{~mm}$ for the Comp 24PM and Comp 4PM, respectively. In Figure 17b, the undesirable upwards bending that occurs during the downstroke due to the contact gaps and flexibility of Delrin is shown. The relative bending deflection between the compliant spar tip marker and the solid spar tip marker is $94.3 \mathrm{~mm}$ and $72.6 \mathrm{~mm}$ for the Comp 24PM and Comp 
4PM, respectively. Overall, the bending deflection of the compliant spines during the upstroke is greater than the bending deflection during the downstroke.

\section{Conclusions and Future Work}

The aforementioned test proved to be successful in producing consistent and repeatable flight data over more than eight free flight flapping cycles. Through these flight tests, the ornithopter body dynamics were shown to be significant, $\pm 4 \mathrm{gs}$ for the solid configuration. Also the peak in the body's vertical acceleration that occurred after the upstroke-downstroke and downstroke-upstroke transition points was attributed to the dynamics of the thrust flap. The effect of the presence of the compliant spines in the wings on the body dynamics and leading edge spar deflection was examined through the flight tests. The compliant spine presence reduced the body's center of mass positive acceleration which translates into overall lift gains. Also inserting the compliant spine into the leading edge spar introduced an asymmetry between the upstroke and the downstroke, as desired. However, the data shows that undesirable bending occurs during the downstroke due to the flexibility of Delrin and the contact gaps. Future work includes comparing the free flight data to previous bench top data. Also during the flight test, electric current drawn by the battery was measured and recorded, thus further data analysis is needed to investigate the effect of the presence of the compliant spine on the vehicle's power consumption. Finally a design modification is recommended to mitigate the downstroke flexibility so that the compliant spine behaves more like the carbon fiber spar during this portion of the flight cycle.

\section{Acknowledgments}

The authors gratefully acknowledge the support of AFOSR grant number FA9550-09-1-0632 and the AFOSR program manager, David Stargel. The computational work needed to design the compliant spines was supported in part through instrumentation funded by the National Science Foundation through grant OCI-0821527. Also the authors are very thankful to the AFRL indoor flight lab team especially Gregory Reich and Gregory Parker for their support during the flight testing. The resources of the NASA Langley Research Center, Pennsylvania State University, the University of Maryland and the Morpheus Lab are also appreciated.

\section{References}

${ }^{1}$ Shyy, W. Berg, M., and Ljungqvist, D. "Flapping and flexible wings for biological and micro air vehicles", Progress in Aerospace Sciences,(1999) 35: 455-505

2 Tobalske, B.W., and Dial, K.P. "Flight kinematics of Black-billed Magpies and pigeons over a wide range of speeds", Journal of Experimental Biology , (1996) 99: 263-280.

3 Tobalske, B.W. "Physiology and Biomechanics of Gait Selection in Flying Birds", Physiol Biochem Zool., (2000) 73(6):736-50.

${ }^{4}$ Fenelon, M.A.A., and Furukawa, T. "Design of an Active Flapping Wing Mechanism and a Micro Aerial Vehicle Using a Rotary Actuator." Mechanism and Machine Theory 45 (2009): 137-46.

${ }^{5}$ McDonald, M., and Sunil K. Agrawal. "Design of a Bio-Inspired Spherical Four-Bar Mechanism for Flapping-Wing Micro Air-Vehicle Applications." Journal of Mechanisms and Robotics, (2010) 021012

${ }^{6}$ Cox, A., Monopoli, D., Goldfarb, M., and Garcia, E. "Development of piezoelectrically actuated micro-aerial vehicles", SPIE Symposium on Microrobotics and Microassembly, vol. 3834, pp. 101-108, September 1999. 
${ }^{7}$ Wissa, A., Tummala, Y., Hubbard Jr., J. E., and Frecker, M. "Passively Morphing Ornithopter Wings using a Novel Compliant Spine: Design and Testing," Smart Materials and Structures (2102) 21 094028

${ }^{8}$ Wissa, A., Tummala Y., Hubbard Jr., J. E., Frecker M., and Brown, A. "Testing of novel compliant spines for passive wing morphing," Proc. Smart Materials, Adaptive Structures and Intelligent Systems Conf. 2011-5198, Scottsdale, AZ

${ }^{9}$ Mueller D., Gerdes J., and Gupta, S.K. "Incorporation of passive wing folding in flapping wing miniature air vehicles." ASME Mechanism and Robotics Conference, August 30-September 2, 2009, San Diego

${ }^{10}$ Billingsley, D., Slipher, G., and Grauer, J., "Testing of a Passively Morphing Ornithopter Wing," AIAA Paper 2009-1828.

${ }^{11}$ Brown, R. H. "The Flight of Birds: Wing Function in Relation to Flight Speed." Diss. University of Cambridge, 1952.

${ }^{12}$ Tummala, Y., Wissa A., Frecker, M., and Hubbard Jr., J. E. "Design of a passively morphing ornithopter wing using a novel compliant spine" Proc. Smart Materials, Adaptive Structures and Intelligent Systems Conf. 2010-3637, Philadelphia PA

${ }^{13}$ Tummala, Y., Wissa, A., Frecker, M., and Hubbard Jr., J. E. " Design optimization of a compliant spine for dynamic applications," Proc. Smart Materials, Adaptive Structures and Intelligent Systems Conf. 2011-5207, Scottsdale, AZ

${ }^{14}$ Harmon, R. "Aerodynamic Modeling of Flapping Membrane Wing Using Motion Tracking Experiments, Aerospace Engineering." Thesis. College Park / University of Maryland, 2009.

${ }^{15}$ Grauer J, Ulrich E, Hubbard J, Pines D, Humbert J S. "Testing and system identification of an ornithopter in longitudinal flight. " Journal of Aircraft, (2011): 48, 660-667. 